\section{Perplexing p38}

\section{By Michael J. Haas, Senior Writer}

Although p38 kinase inhibition had previously been shown to lower necrosis of plaques in atherosclerosis in vitro, the same researchers now have found that inhibiting the target in vivo could instead lead to increased risk of thrombotic events. ${ }^{1}$ Although companies with small molecule p38 inhibitors in Phase II testing to treat atherosclerosis, such as GlaxoSmithKline plc and the partners Bristol-Myers Squibb Co. and Ligand Pharmaceuticals Inc., haven't reported such side effects, the data suggest the potential risk may be inadequately assessed by existing animal models or clinical trial designs.

The findings from Columbia University and University of California, Los Angeles researchers run counter to prior results the Columbia group published in $2005 .^{2}$

Mitogen-activated protein kinase 14 (MAPK14; p38) is one of four p38 isoforms involved in cell proliferation, cell development, transcription and cellular responses to stress such as inflammation.

Although the mechanism of action of p38 inhibitors in atherosclerosis is not fully understood, the rationale for targeting p38 in cardiovascular disease is to block inflammation in endothelial cells at the site of atherosclerotic lesions. This, in turn, is thought to prevent the recruitment of monocytes that are responsible for a positive feedback loop of macrophage differentiation, macrophage apoptosis and plaque formation.

In 2005, the Columbia group had shown that p38 inhibition prevented cholesterol-loaded macrophages from undergoing apoptosis in vitro by blocking activation of DNA-damage-inducible transcript 3 (DDIT3; CHOP10; CHOP; GADD153). CHOP is a protein that plays a role in programmed cell death.

Based on those data, the team hypothesized that p38 inhibition might prevent necrosis of vulnerable plaques, which are characterized by a highly inflammatory state, a soft core and a thin fibrous cap. When macrophages in the core of a vulnerable plaque become necrotic, the plaque can slough off the artery wall and cause a blood clot.

The Columbia researchers then teamed up with a researcher from UCLA to find out whether p38 inhibition could indeed stop vulnerable plaques from dying.

Surprisingly, as reported now in the Journal of Clinical Investigation, they found that mouse models of atherosclerosis with p38-deficient macrophages exhibited greater-not less-plaque necrosis than controls. Plaques in the p38-deficient mice showed signs of advanced atherosclerotic disease such as lower collagen content and thinning of the plaque's fibrous cap.
The results indicated that $\mathrm{p} 38$-deficient macrophages could increase the risk of thrombotic events.

The team also found that p38 deficiency or inhibition in mouse macrophage cultures induced apoptosis by suppressing activation of the prosurvival protein kinase B (PKB; Akt) pathway.

\section{Opaque on plaque}

The team wrote in JCI that it was unclear why the net effect of p38 inhibition was proapoptotic in the in vivo study but prosurvival in the 2005 in vitro study. The researchers concluded that more work would be needed to elucidate the reason.

Tracie Seimon, lead author of both studies, told SciBX that the new data don't derail the development of p38 inhibitors for atherosclerosis just yet. But she thinks the study shows that p38's role in atherosclerosis and plaque necrosis is complex and underscores the need for better models to elucidate it.

"Our work doesn't fully say that p38 inhibition can't be useful for atherosclerosis," she said. "Those inhibitors may also affect nonmacrophage cells, such as endothelial cells," where p38 inhibition is thought to exert an anti-inflammatory effect that slows monocyte recruitment.

Seimon is an investigator in the research group of senior author Ira Tabas, vice chairman for research in medicine and professor of medicine and anatomy and cell biology at Columbia.

Stephen Miller, senior director of translational biology in inflammatory and metabolic diseases at Array BioPharma Inc., agreed that more in vivo work is needed to clarify the complex role p38 plays in atherosclerosis. "As the authors point out, it will be a balance of factors involving p38 in macrophages as well as endothelial cells in the plaques," he said.

Like Seimon, Miller did not think the findings would preclude the use of p38 inhibitors to treat atherosclerosis or other diseases. He noted that the majority of literature to date suggests that such compounds would have no adverse cardiovascular effects.

In about 1,000 patients across all Phase II trials of p38 inhibitors known to him, Miller said, "no obvious cardiovascular safety signal has shown up for those inhibitors. Also, increased risk of cardiovascular events has not appeared in preclinical safety and toxicity studies lasting 6-9 months."

Although Seimon suggested that "imaging of carotid artery plaques in patients in p38 inhibitor trials" might be advisable, Miller noted that this was easier said than done.

"In noncardiovascular indications, it's not a simple thing to track atherosclerosis, because monitoring plaques requires pretty specific imaging techniques and these are not easily added on to a trial design," he said.

Miller also noted that there are no obvious markers to distinguish between vulnerable and stable plaques - of which the latter calcify and become metabolically inactive over time. As a result, Miller said, there is no easy way to determine which patients might be at risk of atherosclerosis-related thrombosis. 


\section{TARGETS \& MECHANISMS}

According to Miller, even in preclinical research there are no accepted animal models of vulnerable plaques. "You'd want to develop a model of vulnerable plaques that mimics human disease, then test p38 inhibitors in those models" to see whether inhibition raises or lowers the risk of atherosclerosis-related thrombosis, he said.

Array has two p38 inhibitors in clinical development. In February, the company began a Phase II trial of ARRY-797 in ankylosing spondylitis, which is a form of chronic arthritis that affects the spine and pelvis. Later this year, the company expects to begin a Phase Ib/IIa trial of ARRY-614 to treat cancer. Both trials should finish in 2010, Miller said.

In addition to Array, at least five other companies have p38 inhibitors in development for inflammatory conditions such as rheumatoid arthritis (RA), chronic obstructive pulmonary disease (COPD) and psoriasis.

The most advanced p38 inhibitors to treat atherosclerosis are GW856553 from GlaxoSmithKline and BMS-582949 from BristolMyers and Ligand. All three companies declined to comment on the JCI findings.

Because she is interested in macrophages generally-not just their role in atherosclerosis-Seimon said her studies are now focusing on the role of p38 in macrophage survival and/or apoptosis in infectious diseases. She suggested that follow-on studies to the experiments published in JCI could examine the effect of p38 inhibition on the size and complexity of plaques in mice with pre-existing atherosclerotic lesions.

"A specific follow-up would be p38 knockout in endothelial cells, to see whether this affects monocyte recruitment to lesions," as this has not yet been shown in animal models, she said.

According to Seimon, the findings reported in JCI are not patented or licensed.

Haas, M.J. SciBX 2(13); doi:10.1038/scibx.2009.518

Published online April 2, 2009

\section{REFERENCES}

1. Seimon, T. et al. J. Clin. Invest.; published online March 16, 2009; doi:10.1172/JCl37262

Contact: Tracie Seimon, Columbia University, New York, N.Y. e-mail: tad2105@columbia.edu

2. DeVries-Seimon, T. et al. J. Cell Biol. 171, 61-73 (2005)

COMPANIES AND INSTITUTIONS MENTIONED

Array BioPharma Inc. (NASDAQ:ARRY), Boulder, Colo. Bristol-Myers Squibb Co. (NYSE:BMY), New York, N.Y Columbia University, New York, N.Y. GlaxoSmithKline plc (LSE:GSK; NYSE:GSK), London, U.K. Ligand Pharmaceuticals Inc. (NASDAQ:LGND), San Diego, Calif. University of California, Los Angeles, Calif. 